\title{
(6) OPEN ACCESS \\ Associations between late and moderately preterm birth and smoking, alcohol, drug use and diet: a population-based case-cohort study
}

\author{
Lucy K Smith, ${ }^{1}$ Elizabeth S Draper, ${ }^{1}$ T Alun Evans, ${ }^{1}$ David J Field, ${ }^{1}$ \\ Samantha J Johnson, ${ }^{1}$ Bradley N Manktelow, ${ }^{1}$ Sarah E Seaton, ${ }^{1}$ Neil Marlow, ${ }^{2}$ \\ Stavros Petrou, ${ }^{3}$ Elaine M Boyle ${ }^{1}$
}

'Department of Health Science, University of Leicester, Leicester, UK

${ }^{2}$ Institute for Womens Health, University College London, London, UK

${ }^{3}$ Clinical Trials Unit, University of Warwick, Coventry, UK

\section{Correspondence to}

Dr Lucy K Smith, Department of Health Sciences, University of Leicester, 22-28 Princess Road West, Leicester LE1 6TP, UK; Iks1@le.ac.uk

Received 29 July 2014 Revised 29 March 2015 Accepted 8 April 2015 Published Online First 13 May 2015

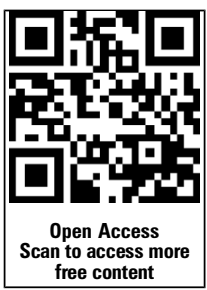

\section{SLinked}

- http://dx.doi.org/10.1136/ fetalneonatal-2015-308768

\section{CrossMark}

To cite: Smith LK, Draper ES, Evans TA, et al. Arch Dis Child Fetal Neonatal Ed 2015;100 F486-F491.

\section{ABSTRACT}

Objective This study explores the associations between lifestyle factors and late and moderate preterm birth (LMPT: $32^{+0}-36^{+6}$ weeks' gestation), a relatively underresearched group.

Study design A population-based case-cohort study was undertaken involving 922 LMPT and 965 term (37+ weeks' gestation) singleton live and stillbirths born between 1 September 2009 and 31 December 2010 to women residing in Leicestershire and Nottinghamshire, UK. Poisson multivariable regression models were fitted to estimate relative risks (RR) of LMPT birth associated with maternal smoking, alcohol and recreational drug use, and diet.

Results Women who smoked during pregnancy were at $38 \%$ increased risk of LMPT birth compared with non-smokers (RR 1.38, 95\% Cl (1.04 to 1.84)). Low consumption of fruit and vegetables was associated with a 31\% increased risk compared with those who reported eating higher consumption levels (RR 1.31 (1.03 to 1.66)). Women who did not have any aspects of a Mediterranean diet were nearly twice as likely to deliver LMPT compared with those whose diet included more Mediterranean characteristics (RR 1.81 (1.04 to 3.14)). Women who smoked and consumed low levels of fruit and vegetables ( $5 \%$ of women) were at particularly high risk ( $R R=1.81$ (1.29 to 2.55)). There was no significant effect of alcohol or recreational drug use on LMPT birth. Conclusions Smoking and poor diet during pregnancy, factors that strongly impact on very preterm birth, are also important at later gestations and experienced together are associated with an elevated rate of risk. Our findings suggest early cessation of smoking during pregnancy may be an effective strategy to reduce LMPT births.

\section{INTRODUCTION}

Internationally, preterm birth is a major public health concern causing over 1 million deaths annually and high rates of morbidity and disability among survivors. ${ }^{1}$ Consequently, prevention of preterm birth has been a major focus of interest. Research has highlighted wide socio-economic inequalities in the rates of preterm birth ${ }^{2}$ though the precise mechanistic links for these inequalities are unknown. Since mortality and morbidity rates are particularly high for those infants born very preterm ( $<32$ weeks' gestation), the impact of lifestyle factors on this group is well documented.

\section{What is already known on this topic?}

The impact of smoking on very preterm birth is well documented, but the effect on late and moderately preterm (LMPT) births is poorly understood.

- There is conflicting evidence regarding the influence of diet and other lifestyle factors on the risk of preterm birth.

\section{What this study adds?}

Smoking during pregnancy is associated with increased risk of LMPT birth.

- Women who give up smoking before pregnancy or during the first trimester have similar risks of LMPT birth to non-smokers, suggesting that cessation by the end of the first trimester may reduce the risk of LMPT birth.

- A Mediterranean diet and a diet high in fruit and vegetables are associated with a lower risk of LMPT birth.

In contrast, late and moderate preterm (LMPT) births (32-36 weeks of gestation) represent $75 \%$ of all preterm births, affecting around 40000 births a year in the UK alone, ${ }^{4}$ yet the impact of lifestyle factors on delivery specifically at LMPT gestations is poorly understood.

Many lifestyle factors are closely linked to material and financial deprivation, and their detrimental effects on preterm birth have been reported. Key risk factors associated with preterm birth include smoking, ${ }^{5}$ excessive alcohol intake ${ }^{6} 7$ and recreational drug use ${ }^{8}$ during pregnancy. The biological mechanisms by which these factors may lead to preterm birth have not been fully elucidated. However, it is plausible that maternal psychological and/or physical stress, which are known to be associated with adverse lifestyle factors, may promote activation of neuroendocrine or proinflammatory pathways in favour of parturition. ${ }^{9}$ There is increasing research exploring the impact of maternal diet on preterm birth, although evidence relating to a Mediterranean-style diet (high levels of fish, fruit and vegetable consumption and low levels of meat 
and coffee consumption) $)^{10-12}$ and a diet high in folate ${ }^{13} 14$ has been inconclusive. Here we use detailed information from a large population-based study to explore the impact of a range of lifestyle factors on LMPT birth.

\section{METHODS}

The Late And Moderately preterm Birth Study (LAMBS) is a prospective, population-based study of all live births and stillbirths $32^{+0}-36^{+6}$ weeks' gestation whose mothers were normally resident in a predefined region of Leicestershire and Nottinghamshire, UK, between 1 September 2009 and 31 December 2010. A term-born comparison group $\left(37^{+0}-42^{+6}\right.$ weeks' gestation) was recruited based on random sampling of the times of birth of babies during the previous year. This method overcomes the bias that may occur if more naive sampling methods were used: for example, selecting the next term birth to occur at the maternity unit after a LMPT birth. Such sampling methods would be likely to result in an oversampling of high-risk term births as these tend to be delivered in the same places and times as LMPT births: for example, no births would have been sampled from the midwifery-led unit. Analyses were restricted to singleton births of consenting families. Gestation was assessed using ultrasound scan in the first trimester $(97.1 \%)$, last menstrual period or ultrasound scan later in pregnancy $(2.2 \%)$ or clinical assessment at birth $(0.7 \%)$.

Box 1 details the categorisation of lifestyle and sociodemographic factors. Information on smoking and alcohol use at preconception, early pregnancy (1-13 weeks gestation' (first trimester)) and later pregnancy (14-32 weeks' gestation) was obtained via a maternal interview shortly after delivery. Recreational drug use during pregnancy was collected as a binary variable. Diet was explored using (1) low vegetable and fruit intake and (2) lack of all aspects of a Mediterranean diet. The use of folic acid supplements during pregnancy was also recorded.

Information was obtained from the maternal interview on factors of known clinical relevance that might confound the relationship with LMPT birth: highest level of educational achievement, maternal age and mother's ethnic group. Body mass index (BMI) was based on mother's weight pre-pregnancy/ at booking recorded in the maternal notes.

Data were analysed as a case-cohort design, ${ }^{15}$ including all the LMPT births and weighting the random sample of term births to replicate all term births in the population allowing the calculation of relative risks (RRs). Each term birth was assigned a design weight based on the total number of 37-42 weeks' gestation births in the region $(\mathrm{n}=20023)$ and divided by its probability of inclusion in the sampling frame $(n=1292$; weight $=15$ ). Weighted univariable analyses were undertaken to explore the relationship between each lifestyle factor and LMPT birth, where the sampled subjects are weighted with the inverses of their inclusion probabilities in the sample. Weighted Poisson multivariable regression models were used to examine the independent effects of these factors on LMPT birth, quantified using estimated RRs and 95\% CIs before and after adjusting for maternal age, ethnicity, BMI and educational level. Robust SEs were used to calculate CIs.

\section{RESULTS}

In total, 938 singleton LMPT births (83\%) and 982 singleton term births (76\%) were recruited (6.0 LMPT births per 100 births >32 weeks' gestation). Thirty births (1.6\%) were excluded due to missing information (922 LMPT and 968 term births).

\section{Box 1 Classification of lifestyle and demographic factors}

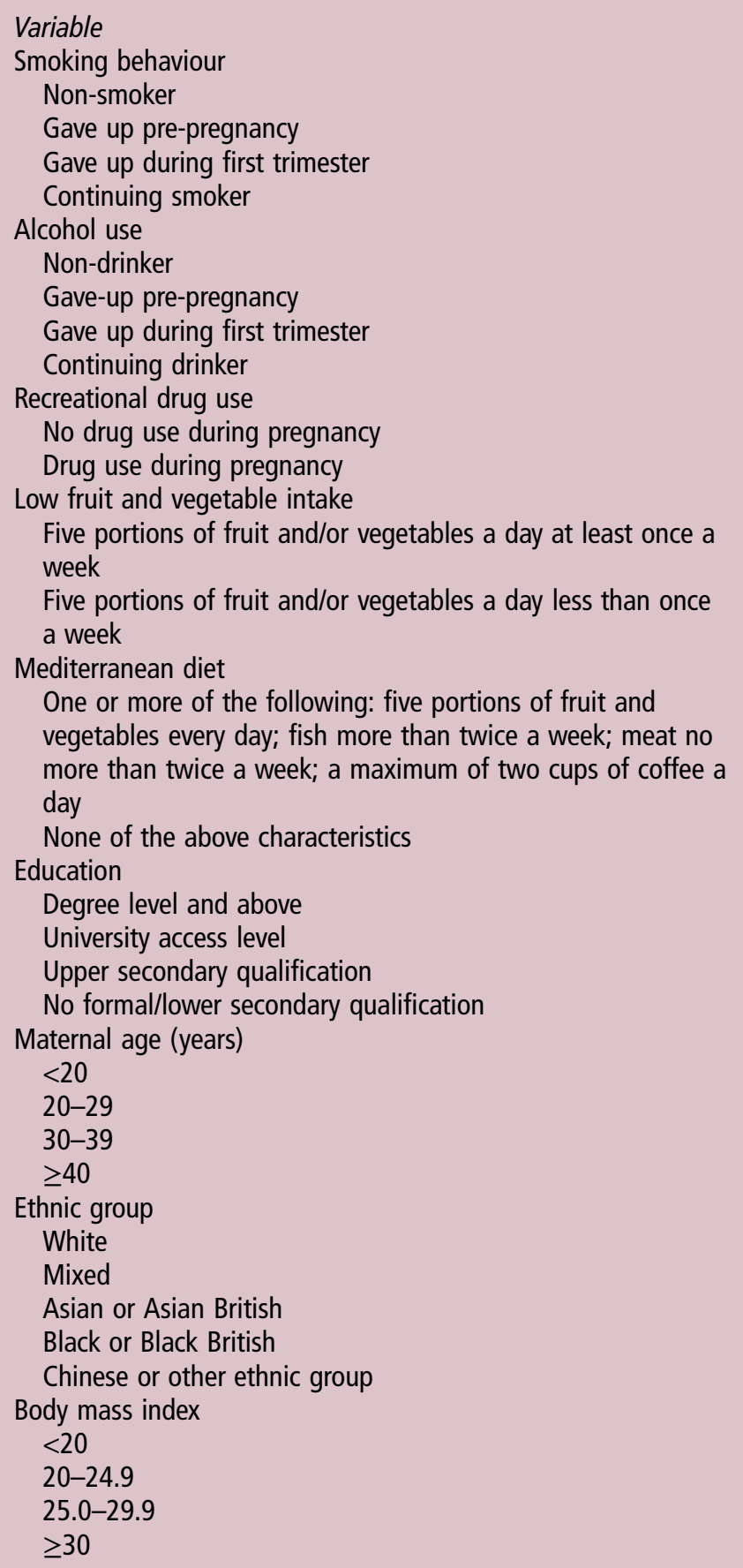

Table 1 shows the percentage and rate of LMPT births for each lifestyle factor for the univariable analysis. Fifty-eight per cent of women had never smoked regularly; $18 \%$ gave up before pregnancy; $6 \%$ gave up in the first trimester and $18 \%$ continued to smoke. Non-smokers and those who gave up smoking before pregnancy or in the first trimester had similar rates of LMPT birth (5.8/1000 births, 4.7/1000 births and 6.3/ 1000 births, respectively), while continuing smokers had significantly higher rates (8.4/1000 births). In contrast, the highest rates of LMPT birth were in those women who never drank alcohol (7.0/1000 births). Rates were lower for those women who gave up alcohol before pregnancy, gave up in the first 
Table 1 Numbers of late and moderately preterm (LMPT) and term births and rates and univariable relative risk of LMPT birth by maternal lifestyle characteristics

\begin{tabular}{|c|c|c|c|c|c|c|}
\hline Indicator & LMPT births & Term-born births & $\begin{array}{l}\text { Rate of LMPT births/ } \\
100 \text { births weighted* }\end{array}$ & $\begin{array}{l}\text { Relative risk } \\
\text { of LMPT birth }\end{array}$ & $95 \% \mathrm{Cl}$ & $P$ value \\
\hline \multicolumn{7}{|c|}{ Use of recreational drugs during pregnancy } \\
\hline No & 903 & 956 & 5.9 & 1 & - & 0.156 \\
\hline Yes & 19 & 12 & 9.6 & 1.61 & 0.83 to 3.13 & \\
\hline \multicolumn{7}{|l|}{ Smoking status } \\
\hline Non-smoker & 524 & 572 & 5.8 & 1 & - & 0.001 \\
\hline Gave-up before pregnancy & 145 & 196 & 4.7 & 0.82 & 0.65 to 1.03 & \\
\hline Gave-up in 1st trimester & 57 & 57 & 6.3 & 1.09 & 0.76 to 1.56 & \\
\hline Smoker during pregnancy & 196 & 143 & 8.4 & 1.46 & 1.16 to 1.83 & \\
\hline \multicolumn{7}{|l|}{ Alcohol use } \\
\hline Non-drinker & 289 & 255 & 7.0 & 1 & - & 0.087 \\
\hline Gave up before pregnancy & 267 & 298 & 5.6 & 0.80 & 0.64 to 1.00 & \\
\hline Gave up in first trimester & 201 & 241 & 5.3 & 0.75 & 0.59 to 0.95 & \\
\hline Drank during pregnancy & 165 & 174 & 6.0 & 0.85 & 0.66 to 1.09 & \\
\hline \multicolumn{7}{|c|}{ Daily consumption of five fruit and vegetables } \\
\hline At least once a week & 728 & 819 & 5.6 & 1 & - & 0.001 \\
\hline Never & 194 & 149 & 8.0 & 1.43 & 1.15 to 1.78 & \\
\hline \multicolumn{7}{|l|}{ Mediterranean diet factors } \\
\hline$\geq 1$ & 889 & 951 & 5.9 & 1 & - & 0.013 \\
\hline None & 33 & 17 & 11.5 & 1.96 & 1.15 to 3.32 & \\
\hline
\end{tabular}

trimester or drank throughout pregnancy (5.6/1000 births, 5.3/ 1000 births, 6.0/1000 births, respectively). Women using recreational drugs had a higher rate of LMPT birth (9.6/1000 births) than those who did not (5.9/1000 births) but numbers were small $(n=31)$ and not significant $(p=0.156)$. Eighteen per cent of women ate five portions of fruit and vegetables a day less than once a week, and this was significantly associated with higher rates of LMPT birth (8.0/1000 births) compared with those eating fruit and vegetables more frequently $(5.6 / 1000$ births) $\quad(\mathrm{p}=0.001)$. Only $2 \%$ of women lacked all Mediterranean diet characteristics, but this was strongly associated with LMPT birth (11.5/1000 births vs 5.9/1000 births for the rest of the population).

An initial multivariable model was developed (table 2) that solely included the lifestyle factors followed by a further model adjusting for maternal age, educational level, BMI and ethnicity. Adjustment showed little change in the RRs apart from alcohol consumption. Based on the fully adjusted model, women who smoked during pregnancy were at 38\% higher risk of LMPT birth than those who had never smoked regularly (adjusted RR (ARR) 1.38 (1.04to 1.84)). There was a non-significant doseresponse relationship for level of smoking during pregnancy between light smokers ( $\leq 10$ cigarettes per day) and moderate/ heavy smokers $(>10$ cigarettes a day) $(p=0.707)$. There was no evidence of an increased risk of LMPT birth for those women who gave up smoking before or during early pregnancy (ARR of 0.93 and 1.12 , respectively).

Eating five portions of fruit and vegetables a day less than once a week was associated with an RR of 1.31 for LMPT birth compared with those women who eat fruit and vegetables more frequently (ARR 1.31 (1.03 to 1.66)). Few women reported not having any of the characteristics of a Mediterranean diet at only 2.6\%; however, their risk was considerably greater being almost twice as likely to have a LMPT birth than women with at least one characteristic of a Mediterranean diet (ARR 1.81 (1.04 to 3.14)).
In the initial multivariable model, women who had never drunk alcohol were at increased risk of LMPT birth compared with women who had drunk alcohol before or during pregnancy. However, this was reversed after adjustment for ethnicity, although neither estimate was statistically significant. This change was due to higher risk of LMPT birth among Asian women who were more likely to be non-drinkers. Of those women who had never drunk alcohol, $43 \%$ were Asian or Asian British compared with $6 \%$ of women who had drunk alcohol before or during their pregnancy being Asian or Asian British. Consequently, ethnicity was a confounder via its relationship with alcohol consumption.

At particularly high risk of LMPT birth were women who were both smokers during pregnancy and ate five portions of fruit and vegetables a day less than once a week (1 in 17), whose additive risk of LMPT birth was greatly increased at $81 \%$ higher than women who were not regular smokers and who also ate five a day more regularly (ARR 1.81 (1.29 to 2.55)). As highlighted previously, there was no multiplicative interaction effect between these factors. Interactions between each of the lifestyle effects were explored but none were statistically significant $(\mathrm{p}>0.1)$.

Sensitivity analyses showed little difference in the effect of lifestyle factors on those LMPT births where the onset of labour was spontaneous compared with other LMPT births, suggesting that the impact of lifestyle factors was qualitatively similar for both groups. Further analyses to assess whether folic acid supplementation in the first trimester explained the effect of fruit and vegetable consumption showed negligible effect on the RR estimate for fruit and vegetable consumption (ARR $1.30(1.02$ to 1.65$)$ ), and a negative effect of folic acid intake (ARR 1.26 (0.98 to 1.61$)$ ), that is, women who used folic acid supplementation had a higher risk of LMPT birth than those who reported no supplementation although this was not significant $(p=0.071)$. A sensitivity analysis to assess whether the relationship between lack of a Mediterranean diet and LMPT birth was 
Table 2 Relative risks of late and moderately preterm (LMPT) birth for lifestyle factors from multivariable model, before and after including mother's age, ethnicity, body mass index (BMI) and educational level

\begin{tabular}{|c|c|c|c|c|c|c|}
\hline \multirow[b]{3}{*}{ Indicator } & \multicolumn{6}{|c|}{ Risk of late and moderate preterm birth } \\
\hline & \multicolumn{3}{|c|}{ Model including lifestyle factors } & \multicolumn{3}{|c|}{$\begin{array}{l}\text { Model including lifestyle factors adjusted for } \\
\text { socio-demographic factors }\end{array}$} \\
\hline & Relative risk & $95 \% \mathrm{Cl}$ & $p$ Value & Relative risk & $95 \% \mathrm{Cl}$ & $\mathrm{p}$ Value \\
\hline \multicolumn{7}{|l|}{ Lifestyle factors } \\
\hline \multicolumn{7}{|l|}{ Use of recreational drugs during pregnancy } \\
\hline No & 1 & - & 0.486 & 1 & - & 0.495 \\
\hline Yes & 1.27 & 0.65 to 2.51 & & 1.27 & 0.64 to 2.50 & \\
\hline \multicolumn{7}{|l|}{ Smoking status } \\
\hline Non-smoker & 1 & - & 0.027 & 1 & - & 0.082 \\
\hline Gave up before pregnancy & 0.86 & 0.67 to 1.09 & & 0.93 & 0.72 to 1.20 & \\
\hline Gave up in first trimester & 1.07 & 0.73 to 1.56 & & 1.12 & 0.76 to 1.66 & \\
\hline Smoker during pregnancy & 1.35 & 1.05 to 1.72 & & 1.38 & 1.04 to 1.84 & \\
\hline \multicolumn{7}{|l|}{ Alcohol use } \\
\hline Non-drinker & 1 & - & 0.205 & 1 & - & 0.918 \\
\hline Gave up before pregnancy & 0.83 & 0.66 to 1.04 & & 1.02 & 0.78 to 1.34 & \\
\hline Gave up in first trimester & 0.78 & 0.61 to 0.99 & & 0.98 & 0.73 to 1.31 & \\
\hline Drank during pregnancy & 0.86 & 0.66 to 1.13 & & 1.08 & 0.79 to 1.48 & \\
\hline \multicolumn{7}{|l|}{ Daily consumption of five fruit and vegetables } \\
\hline At least once a week & 1 & - & 0.022 & 1 & - & 0.027 \\
\hline Never & 1.31 & 1.04 to 1.64 & & 1.31 & 1.03 to 1.66 & \\
\hline \multicolumn{7}{|l|}{ Mediterranean diet factors } \\
\hline$\geq 1$ & 1 & - & 0.052 & 1 & - & 0.036 \\
\hline None & 1.71 & 1.00 to 2.92 & & 1.81 & 1.04 to 3.14 & \\
\hline \multicolumn{7}{|l|}{ Socio-demographic factors } \\
\hline \multicolumn{7}{|l|}{ Mother's age (years) } \\
\hline$<20$ & & & & 1 & - & 0.585 \\
\hline $20-29$ & & & & 1.31 & 0.86 to 1.99 & \\
\hline $30-39$ & & & & 1.37 & 0.88 to 2.13 & \\
\hline $40+$ & & & & 1.32 & 0.71 to 2.45 & \\
\hline \multicolumn{7}{|l|}{ Mother's educational level } \\
\hline Degree level and above & & & & 1 & - & 0.052 \\
\hline University-access level & & & & 1.29 & 1.00 to 1.66 & \\
\hline Upper secondary qualification & & & & 1.36 & 1.06 to 1.76 & \\
\hline No formal/lower secondary qualification & & & & 1.42 & 1.06 to 1.89 & \\
\hline \multicolumn{7}{|l|}{ Mother's ethnicity } \\
\hline White & & & & 1 & - & 0.025 \\
\hline Mixed & & & & 1.70 & 0.97 to 2.99 & \\
\hline Asian or Asian British & & & & 1.54 & 1.13 to 2.11 & \\
\hline Black or Black British & & & & 1.36 & 0.89 to 2.08 & \\
\hline Chinese or other ethnic group & & & & 1.99 & 0.87 to 4.55 & \\
\hline \multicolumn{7}{|l|}{ Mother's BMI } \\
\hline $20.0-24.9$ & & & & 1 & - & 0.001 \\
\hline$<20$ & & & & 1.12 & 0.75 to 1.67 & \\
\hline $25.0-29.9$ & & & & 0.80 & 0.64 to 1.00 & \\
\hline$\geq 30$ & & & & 0.58 & 0.45 to 0.76 & \\
\hline No pre-pregnancy weight recorded & & & & 1.07 & 0.57 to 2.02 & \\
\hline
\end{tabular}

predominantly an effect of fruit and vegetable intake, leaving the indicator for fruit and vegetable intake out of the Mediterranean diet classification, reduced the RR slightly to 1.57 (0.97 to 2.55) but showed no change in the RR for low fruit and vegetable intake. This suggested that it was a combination of a lack of all of the factors in the Mediterranean diet that was related to higher rates of LMPT birth.

\section{DISCUSSION}

Our analysis showed that women who smoked during pregnancy, who ate five portions of fruit and vegetables a day less than once a week or who did not follow any aspects of a Mediterranean diet had the highest rates of LMPT birth. This suggests that these risk factors for very preterm birth persist at later gestations.

A major strength of the LAMBS study was the detailed individual-level data on lifestyle and socio-demographic factors within a large cohort allowing exploration of the relative impact of a range of health behaviours on LMPT birth. Region-wide data on gestational age at birth was available to calculate rates of LMPT birth, and the reliability of gestational age was maximised through use of ultrasound examination data rather than last 
menstrual period that can result in overestimation of preterm birth rates.

Information was available only for women who consented to take part. Kramer et al ${ }^{16}$ suggested that low levels of socioeconomic disparities in preterm birth in their work were related to selection bias, with recruits being more likely to be 'psychologically invested in their pregnancies'. While the percentage of women who consented in LAMBS was high (79\%), response was poorer in the more deprived areas (75\%) compared with the least deprived areas (85\%), leading to underestimation of lifestyle effects. Lifestyle factors were collected after birth, so women who delivered early may reflect more on whether their behaviours may have led to early onset of labour. We relied on self-reported general measures of dietary intake and cigarette smoking rather than detailed food diaries or biochemical measures, so they are, as such, imperfect measures and bias in these methods may have affected the observed associations between lifestyle and LMPT birth.

We included all LMPT births irrespective of whether it followed spontaneous preterm labour or a planned early intervention because of some ongoing health problem. However, analysing only those births following spontaneous preterm labour showed an extremely similar pattern of lifestyles that appeared to predispose to LMPT birth. We chose not to include previous obstetric and medical history in the analyses as we felt that these factors were on the underlying causal pathway between smoking diet and alcohol and preterm birth, hence accounting for them in the model would obscure exploration of behavioural factors.

\section{INTERPRETATION}

The similarity in rates of LMPT birth between non-smokers and women who gave up smoking before pregnancy or in the first trimester suggests two possible explanations. First, women who gave up early in pregnancy may have differed from women who continued to smoke, being more 'psychologically invested' in their pregnancies ${ }^{16}$ and their willingness to quit smoking may be associated with other health-related behaviours during pregnancy associated with improved perinatal outcomes. Women with lower exposure to tobacco and higher education levels have been shown to be more likely to give up smoking during pregnancy. ${ }^{17}$ Alternatively, the risk of smoking may be predominantly during late pregnancy, which would indicate that early smoking cessation reduces their risk of LMPT birth. This conclusion is supported by findings from studies such as the Generation R study, ${ }^{5}$ for all preterm births $<37$ weeks, and suggests that public health strategies should aim for complete smoking cessation rather than reducing the number of cigarettes to improve pregnancy outcomes.

Evidence on the protective effect of a Mediterranean style diet on preterm birth has been inconclusive. We have shown that women who had no characteristics of a Mediterranean diet had nearly double the risk of LMPT birth. However, $<2 \%$ of women were in this group, and consequently, interventions to improve dietary patterns may have a relatively small impact on LMPT rates. In contrast, $16 \%$ of women had low fruit and vegetable intake, and these women had rates of LMPT birth that were nearly one-third higher. Our findings support recent evidence that increasing regular consumption of healthy food is more important than focusing on reducing consumption of unhealthy food. ${ }^{12} \mathrm{~A}$ dramatic reduction in risk of preterm birth with folate supplementation has been shown by Bukowski et al, ${ }^{18}$ but others have failed to replicate this. ${ }^{19}{ }^{20}$ Although fruit and vegetable intake is often regarded as a marker of dietary folate intake, the inclusion of information on supplemental intake of folic acid in the multivariable model resulted in negligible change in the RR for fruit and vegetable consumption. This suggests that this effect may not reflect folate intake specifically but may be indicative of a generally poor diet. Alternatively, it may suggest that dietary folate has a greater protective effect than supplementation.

Our adjusted model confirmed previous studies of no significant relationship between alcohol consumption during pregnancy and LMPT birth ${ }^{21}{ }^{22}$ but is in contrast to a recent systematic review ${ }^{23}$ and two large European studies. ${ }^{24}$ This latter finding may be due to the confounding effect of ethnicity identified here with Asian women, who have a higher rate of LMPT birth, also being less likely to drink alcohol for cultural reasons.

Adjusting our findings for maternal age, ethnicity, BMI and educational level did not lead to any change in the interpretation of the model. This suggests that these effects do not appear to be confounded by these socio-demographic factors suggesting a clear public health message.

\section{CONCLUSIONS}

LMPT births represent the majority of births at preterm gestations. Interventions to reduce LMPT delivery could have a large impact in reducing these 40000 births annually in the UK. Chang et al's ${ }^{1}$ recent review of potential reductions in prematurity highlights smoking cessation as the main lifestyle-related intervention to reduce preterm birth, predicting a reduction of approximately $0.01 \%$. Based on our research, smoking cessation early in pregnancy could have an additional impact on reducing LMPT births. Similarly, an emphasis on increasing regular consumption of healthy food, such as following a Mediterranean diet and higher intake of fruit and vegetables, needs to remain as a key strategy in early pregnancy. However, our findings highlight the urgent need for research into underlying mechanisms of LMPT births in order that cost-effective interventions that reduce these births can be better planned and targeted.

Acknowledgements We thank the members of the Infant Mortality and Morbidity Studies team (University of Leicester) for helpful advice and comments throughout the study.

Contributors DJF, EMB, ESD, BNM, LKS, NM and SP conceived the study idea and designed the study. LKS analysed and interpreted the data with help from BNM, SES and TAE. EMB, DJF, ESD, SJJ, NM and SP contributed to interpretation of the data. LKS wrote a first draft of the manuscript, and all co-authors critically revised the manuscript and approved the final manuscript for submission. The guarantor is LKS.

Funding This article presents independent research funded by the National Institute for Health Research (NIHR) under its Programme Grants for Applied Research (PGfAR) Programme (grant reference number RP-PG-0407-10029). The views expressed are those of the authors and not necessarily those of the NHS, the NIHR or the Department of Health.

Competing interests NM receives a proportion of funding from the Department of Health's NIHR Biomedical Research Centres funding scheme at UCLH/UCL.

Ethics approval The study was approved by the Derbyshire NHS Research Ethics Committee (ref 09/H0401/25; 05/05/2009).

Provenance and peer review Not commissioned; externally peer reviewed.

Open Access This is an Open Access article distributed in accordance with the Creative Commons Attribution Non Commercial (CC BY-NC 4.0) license, which permits others to distribute, remix, adapt, build upon this work non-commercially, and license their derivative works on different terms, provided the original work is properly cited and the use is non-commercial. See: http://creativecommons.org/ licenses/by-nc/4.0/

\section{REFERENCES}

1 Chang $\mathrm{HH}$, Larson J, Blencowe $\mathrm{H}$, et al. Preventing preterm births: analysis of trends and potential reductions with interventions in 39 countries with very high human development index. Lancet 2013;381:223-34.

2 Smith LK, Draper ES, Manktelow BN, et al. Socioeconomic inequalities in very preterm birth rates. Arch Dis Child Fetal Neonatal Ed 2007;92:F11-14. 
3 March of Dimes. White paper on preterm birth: the global and regional toll. White Plains, NY: March of Dimes, 2009.

4 Moser K, Macfarlane A, Chow YH, et al. Introducing new data on gestation-specific infant mortality among babies born in 2005 in England and Wales. Health Stat Q 2007;(35):13-27.

5 Jaddoe VW, Troe EJ, Hofman A, et al. Active and passive maternal smoking during pregnancy and the risks of low birthweight and preterm birth: the Generation $\mathrm{R}$ Study. Paediatr Perinat Epidemiol 2008;22:162-71.

6 Ernhart $\mathrm{CB}$, Wolf AW, Linn PL, et al. Alcohol-related birth defects: syndromal anomalies, intrauterine growth retardation, and neonatal behavioral assessment. Alcohol Clin Exp Res 1985;9:447-53.

7 Sokol RJ, Janisse JJ, Louis JM, et al. Extreme prematurity: an alcohol-related birth effect. Alcohol Clin Exp Res 2007:31:1031-7.

8 Phupong V, Darojn D. Amphetamine abuse in pregnancy: the impact on obstetric outcome. Arch Gynecol Obstet 2007;276:167-70.

9 Wadhwa PD, Culhane JF, Rauh V, et al. Stress, infection and preterm birth: a biobehavioural perspective. Paediatr Perinat Epidemiol 2001;15(Suppl 2):17-29.

10 Mikkelsen TB, Osterdal ML, Knudsen VK, et al. Association between a Mediterranean-type diet and risk of preterm birth among Danish women: a prospective cohort study. Acta Obstet Gynecol Scand 2008;87:325-30.

11 Haugen M, Meltzer HM, Brantsaeter AL, et al. Mediterranean-type diet and risk of preterm birth among women in the Norwegian Mother and Child Cohort Study (MoBa): a prospective cohort study. Acta Obstet Gynecol Scand 2008;87:319-24.

12 Englund-Ogge L, Brantsaeter AL, Sengpiel V, et al. Maternal dietary patterns and preterm delivery: results from large prospective cohort study. BMJ 2014;348:g1446.

13 Khoury J, Haugen G, Tonstad S, et al. Effect of a cholesterol-lowering diet during pregnancy on maternal and fetal Doppler velocimetry: the CARRDIP study. Am J Obstet Gynecol 2007;196:549.e1-7.

14 Khoury J, Henriksen T, Christophersen B, et al. Effect of a cholesterol-lowering diet on maternal, cord, and neonatal lipids, and pregnancy outcome: a randomized clinical trial. Am J Obstet Gynecol 2005;193:1292-301.
15 Onland-Moret NC, van der Schouw YT, Buschers W, et al. Analysis of case-cohort data: a comparison of different methods. J Clin Epidemiol 2007;60:350-5.

16 Kramer MS, Wilkins R, Goulet L, et al. Investigating socio-economic disparities in preterm birth: evidence for selective study participation and selection bias. Paediatr Perinat Epidemiol 2009;23:301-9.

17 Vaz LR, Leonardi-Bee J, Aveyard $\mathrm{P}$, et al. Factors associated with smoking cessation in early and late pregnancy in the smoking, nicotine, and pregnancy trial: a trial of nicotine replacement therapy. Nicotine Tob Res 2014;16:381-9.

18 Bukowski R, Malone FD, Porter FT, et al. Preconceptional folate supplementation and the risk of spontaneous preterm birth: a cohort study. PLoS Med 2009;6: e1000061.

19 Sengpiel V, Bacelis J, Myhre R, et al. Folic acid supplementation, dietary folate intake during pregnancy and risk for spontaneous preterm delivery: a prospective observational cohort study. BMC Pregnancy Childbirth 2013;13:160.

20 Shaw GM, Carmichael SL, Yang W, et al, National Birth Defects Prevention Study. Periconceptional intake of folic acid and food folate and risks of preterm delivery. Am J Perinatol 2011;28:747-52.

21 Cooper DL, Petherick ES, Wright J. The association between binge drinking and birth outcomes: results from the Born in Bradford cohort study. J Epidemiol Community Health 2013;67:821-8.

22 McCarthy FP, O'Keeffe LM, Khashan AS, et al. Association between maternal alcohol consumption in early pregnancy and pregnancy outcomes. Obstet Gynecol 2013;122:830-7.

23 Patra J, Bakker $\mathrm{R}$, Irving $\mathrm{H}$, et al. Dose-response relationship between alcohol consumption before and during pregnancy and the risks of low birthweight, preterm birth and small for gestational age (SGA)-a systematic review and meta-analyses. BJOG 2011;118:1411-21.

24 Pfinder $M$, Kunst $A E$, Feldmann $R$, et al. Preterm birth and small for gestational age in relation to alcohol consumption during pregnancy: stronger associations among vulnerable women? Results from two large Western-European studies. BMC Pregnancy Childbirth 2013;13:49. 